\title{
Thrombin-initiated platelet activation in vivo is vWF independent during thrombus formation in a laser injury model
}

Christophe Dubois, Laurence Panicot-Dubois, Justin F. Gainor, Barbara C. Furie, and Bruce Furie

Division of Hemostasis and Thrombosis, Department of Medicine,

Beth Israel Deaconess Medical Center and Harvard Medical School, Boston, Massachusetts, USA.

\begin{abstract}
Adhesion of platelets to an injured vessel wall and platelet activation are critical events in the formation of a thrombus. Of the agonists involved in platelet activation, thrombin, collagen, and vWF are known to induce in vitro calcium mobilization in platelets. Using a calcium-sensitive fluorochrome and digital multichannel intravital microscopy to image unstimulated and stimulated platelets, calcium mobilization was monitored as a reporter of platelet activation (as distinct from platelet accumulation) during thrombus formation in live mice. In the absence of $\mathrm{VWF}$, platelet activation was normal, but platelet adherence and aggregation were attenuated during thrombus formation following laser-induced injury in the cremaster muscle microcirculation. In WT mice treated with lepirudin, platelet activation was blocked, and platelet adherence and aggregation were inhibited. The kinetics of platelet activation and platelet accumulation were similar in $F c R \gamma^{-/-}$mice lacking glycoprotein VI (GPVI), GPVI-depleted mice, and WT mice. Our results indicate that the tissue factor-mediated pathway of thrombin generation, but not the collagen-induced GPVI-mediated pathway, is the major pathway leading to platelet activation after laser-induced injury under the conditions employed. In the tissue factor-mediated pathway, $\mathrm{VWF}$ plays a role in platelet accumulation during thrombus formation but is not required for platelet activation in vivo.
\end{abstract}

\section{Introduction}

Adhesion and aggregation of platelets to an injured vessel wall are critical steps during thrombus formation. Once platelets become adherent, they are activated and recruit additional circulating platelets, leading to the formation of a thrombus (for review, see refs. 1-3). At high shear rates, vessel wall-associated vWF binds to the platelet receptor glycoprotein Ib (GPIb). This interaction establishes transient binding of the platelet to the vessel wall and immobilizes the platelets at the site of injury $(4,5)$. At physiologic shear rates, both GPIb/V/IX and the integrin $\alpha_{\mathrm{II}} \beta_{3}$ participate in forming intercellular tethers among platelets, leading to the formation of stable platelet aggregates (6). The binding of vWF to GPIb also induces platelet activation, as demonstrated by intracellular calcium mobilization, and leads to the activation of the integrin $\alpha_{\text {IIb }} \beta_{3}$ on the platelet surface $(7,8)$. After this first adhesion step, receptor-ligand interactions synergistically promote stable platelet adhesion. Among them, the binding of GPVI with collagen, integrin $\alpha_{2} \beta_{1}$ with collagen or fibrinogen, and the integrin $\alpha_{\mathrm{IIb}} \beta_{3}$ with fibrinogen or vWF lead to stable platelet accumulation in vitro.

In contrast to results from in vitro experiments where vWF was shown to be essential for initial platelet adhesion, platelet accumulation and thrombus formation were markedly attenuated but not absent in in vivo studies using $\nu W^{-/-}$mice and the ferric chloride model of thrombosis $(9,10)$. Given the central role of vWF in initial calcium mobilization in platelets established in in vitro studies (11), we have examined the role of vWF in vivo in platelet activation by directly monitoring platelet calcium mobilization as

Nonstandard abbreviations used: $\left[\mathrm{Ca}^{2+}\right]_{\mathrm{i}}$, intracellular $\mathrm{Ca}^{2+}$ concentration; GPIb, glycoprotein Ib.

Conflict of interest: The authors have declared that no conflict of interest exists. Citation for this article: J. Clin. Invest. 117:953-960 (2007). doi:10.1172/JCI30537. a reporter of platelet activation; and by monitoring platelet accumulation during thrombus formation in a living animal using multichannel intravital digital fluorescence microscopy. In our model, thrombus formation is initiated by laser-induced vessel wall injury of arterioles within the mouse cremaster microcirculation under specific conditions and parameters. Shear rates in the cremaster arterioles are in the range of 1,400 to $1,600 \mathrm{~s}^{-1}$. The spatial and temporal resolution of 2 features of initial thrombus development can be determined in vivo: (a) platelet activation via monitoring of calcium mobilization in individual platelets; and (b) platelet accumulation, with a peak at about 100 seconds. Using this system, we demonstrate that, under the conditions employed, vWF is not required for platelet activation in vivo, although it does play a role in platelet accumulation during thrombus growth. The thrombin-mediated pathway of platelet activation, as observed in our laser-induced thrombosis model, is independent of VWF, whereas the collagen-mediated pathway of platelet activation, as evaluated using the ferric chloride model of thrombus, may be vWF dependent.

\section{Results}

Incorporation of fura-2-loaded platelets into the developing thrombus. Fura-2/AM is a calcium ion-binding fluorochrome used to measure intracellular calcium mobilization. Fura-2 is characterized by distinct spectral properties in the presence of low and high concentrations of $\mathrm{Ca}^{2+}$. The binding constant, $K_{d}$, of fura- 2 for $\mathrm{Ca}^{2+}$ is $0.14 \mu \mathrm{M}$, and thus this fluorochrome is sensitive to changes in the intracellular $\mathrm{Ca}^{2+}$ concentration $\left(\left[\mathrm{Ca}^{2+}\right]_{\mathrm{i}}\right)$ when, in the resting state, $\mathrm{Ca}^{2+}$ concentrations are significantly lower than $0.14 \mu \mathrm{M}$ while in the activated state, $\mathrm{Ca}^{2+}$ concentrations are significantly higher than $0.14 \mu \mathrm{M}$. $\mathrm{Ca}^{2+}$ binding to fura- 2 results in a shift in the absorbance maximum from $380 \mathrm{~nm}$ to $340 \mathrm{~nm}$. The magnitude of fluo- 

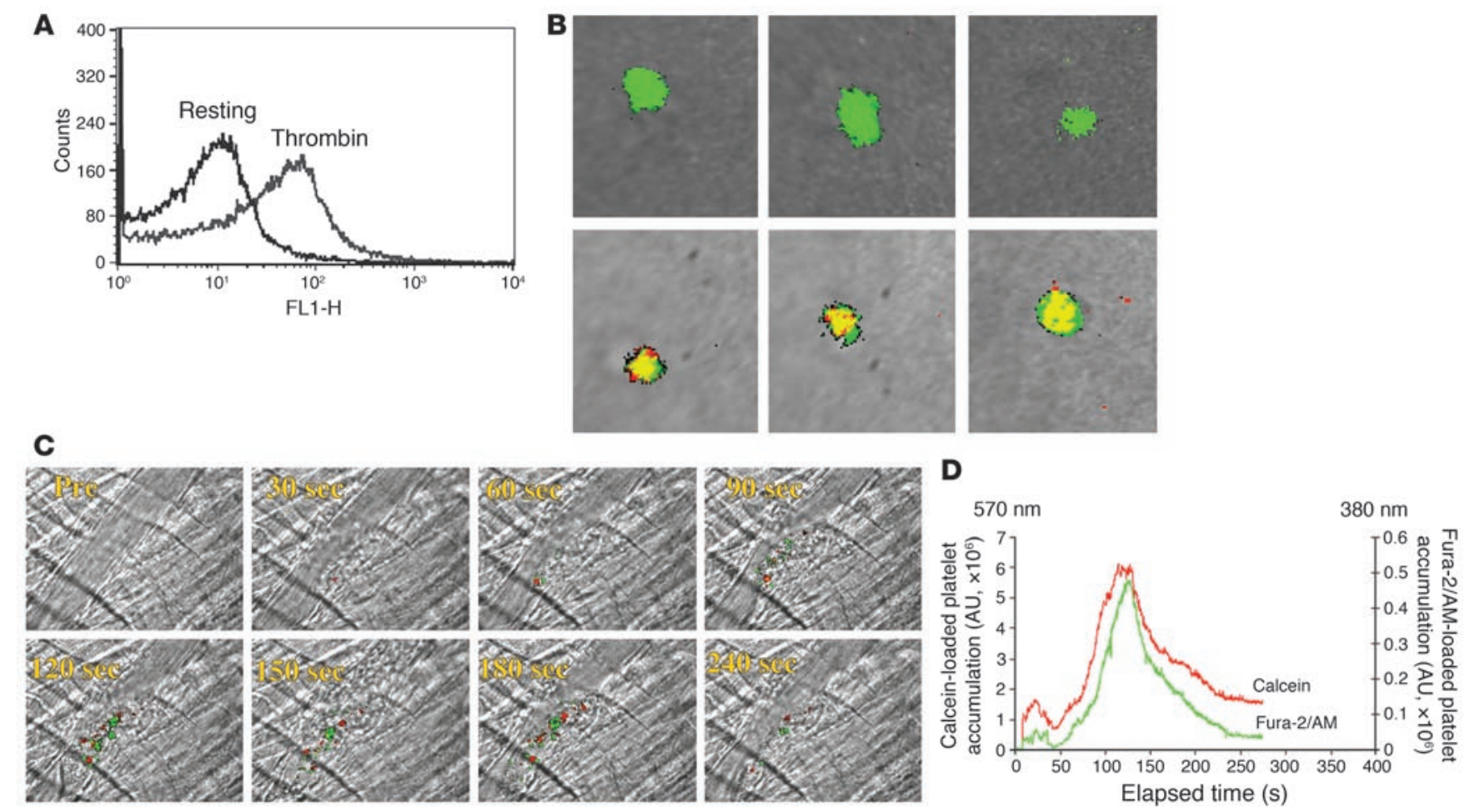

\section{Figure 1}

Properties of fura-2-loaded platelets in vitro and in vivo. (A) P-selectin expression, determined by FACS analysis, on unstimulated or thrombinactivated mouse platelets containing fura-2/AM. (B) Calcium mobilization in unstimulated (top row) or thrombin-activated fura-2-loaded mouse platelets (bottom row) was determined by fluorescence microscopy in vitro. Emission at $510 \mathrm{~nm}$ after excitation at $380 \mathrm{~nm}$ is represented in green, whereas emission at $510 \mathrm{~nm}$ after excitation at $340 \mathrm{~nm}$ is represented in red. Merge is presented in yellow. (C) Platelets loaded with red-orange calcein AM or with fura-2/AM were injected into the circulation of a mouse, and vessel wall injury induced. Images of thrombus formation indicate platelets containing calcein (red) and platelets containing fura-2/AM (green). (D) The median integrated fluorescence intensity of red-orange calcein-labeled platelets or fura-2-labeled platelets in 18 thrombi from 3 mice is plotted as a function of time.

rescence emission at $510 \mathrm{~nm}$ following excitation at $340 \mathrm{~nm}$ and at $380 \mathrm{~nm}$ allows estimation of the $\left[\mathrm{Ca}^{2+}\right]_{i}$. In the current study, we isolated mouse platelets and loaded them with $3 \mu \mathrm{M}$ of fura-2/AM. By flow cytometry, thrombin-activated but not resting fura-2-loaded platelets expressed P-selectin on their surface, assuring that fura-2 did not lead to activation of or interfere with platelet activation (Figure 1A). When resting platelets were excited at $380 \mathrm{~nm}$ and at $340 \mathrm{~nm}$ in vitro, the merged images of these fluorescence emissions observed at $510 \mathrm{~nm}$ were dominated by excitation at $380 \mathrm{~nm}$ (Figure $1 \mathrm{~B}$, top row, green pseudo-color), but no fluorescence emission was observed with excitation at $340 \mathrm{~nm}$ (Figure 1B, top row, red pseudo-color). Merged fluorescence images at $510 \mathrm{~nm}$ following excitation of activated platelets at $380 \mathrm{~nm}$ and at $340 \mathrm{~nm}$ showed that fura- 2 exists in both the $\mathrm{Ca}^{2+}$-bound and $\mathrm{Ca}^{2+}$-free state in these cells, so activated platelets were predominately of the merged yellow pseudo-color (Figure 1B, bottom row).

The efficacy of monitoring platelet recruitment into a developing thrombus using fura- 2 excited at $380 \mathrm{~nm}$ was demonstrated by simultaneously infusing platelets $\left(150 \times 10^{6}\right)$ incubated with either fura-2/AM or with red-orange calcein AM into the circulation of a WT mouse. Ten minutes later, laser-induced arteriole wall injury initiated thrombus formation, and intravital fluorescence and bright-field images were captured with excitation of red-orange calcein at $570 \mathrm{~nm}$ and excitation of fura- 2 at $380 \mathrm{~nm}$ (Figure 1C). These 2 platelet populations exhibited similar kinetics of incorporation into the growing thrombus (Figure 1D). In separate experiments, we infused donor platelets $\left(150 \times 10^{6}\right.$ platelets $)$ loaded with both fura-2/AM and red-orange calcein AM into the circulation of a WT mouse and observed that fluorescent signals corresponding to red-orange calcein and fura- 2 excited at $380 \mathrm{~nm}$ colocalized within the developing thrombus (data not shown). We conclude that platelets containing fura-2/AM have normal platelet function within the context of platelet incorporation into a developing thrombus and that fura-2/AM can be used as an alternative to calcein to monitor the incorporation of platelets into thrombi.

Intracellular calcium status of circulating and thrombus-bound platelets in vivo. We performed intravital wide-field microscopy to visualize fura-2/AM-loaded platelet accumulation in one channel after excitation at $380 \mathrm{~nm}$, calcium mobilization in fura-2/AM-loaded platelets in a second channel after excitation at $340 \mathrm{~nm}$, and the brightfield image in a third channel (Figure 2A). We infused $250 \times 10^{6}$ to $300 \times 10^{6}$ platelets, corresponding to approximately $20 \%$ of the total endogenous platelet number, into a living mouse. All circulating platelets loaded with fura-2/AM had calcium levels characteristic of resting platelets. Fura-2-loaded platelets, monitored by excitation at $380 \mathrm{~nm}$, rapidly bound to the site of laser injury. Calcium mobilization in fura-2-loaded platelets participating in the developing thrombus was detected with excitation at $340 \mathrm{~nm}$ (Figure 2). In WT mice, fura-2-loaded platelets adhered, accumulated, and mobilized intracellular calcium at the site of injury (Figure 2A). In 18 thrombi 
A

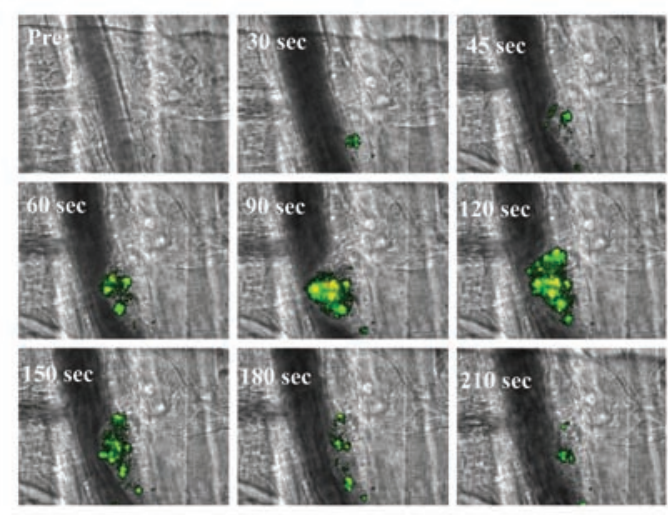

B

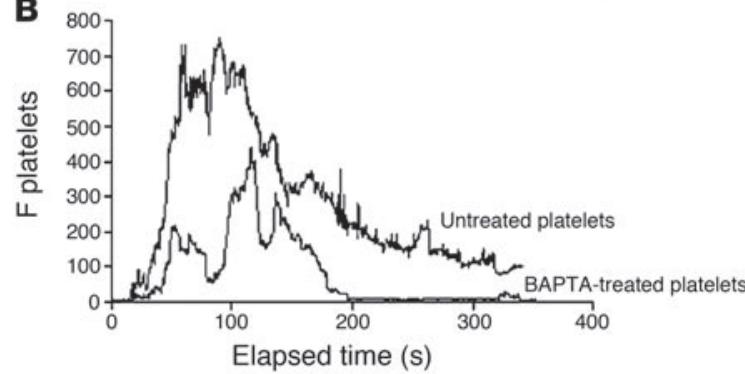

C
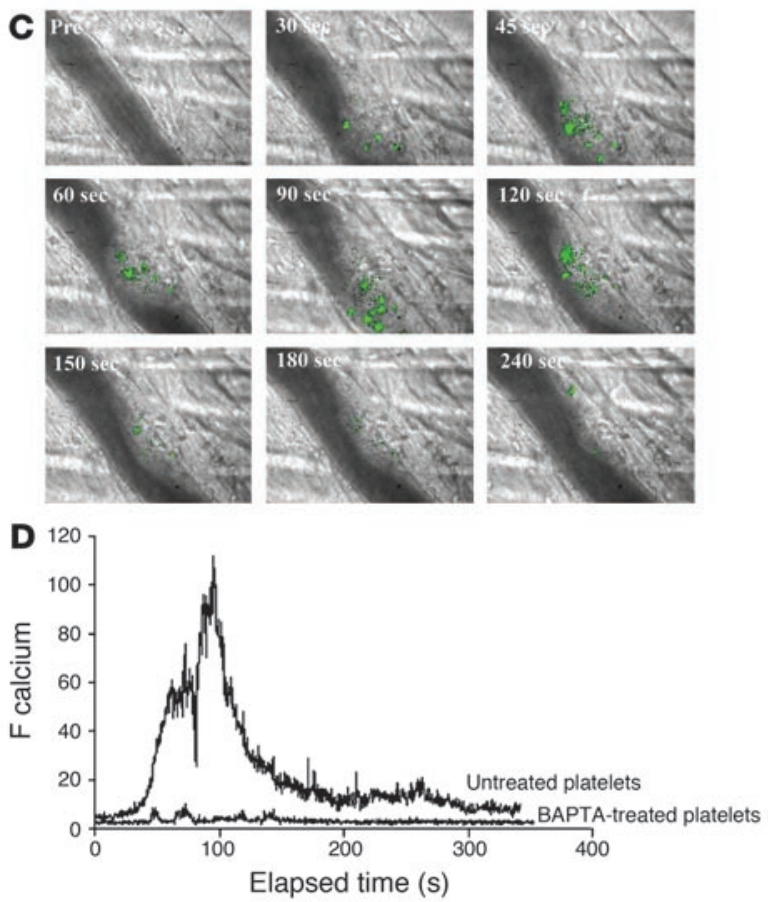

Figure 2

In vivo imaging of calcium mobilization during platelet activation during thrombus formation in WT mice. Platelets isolated from WT mice were loaded with fura-2/AM. Platelets $\left(250 \times 10^{6}\right.$ to $300 \times 10^{6}$ cells) were infused into the circulation of a WT mouse. Following laser-induced vessel wall injury, thrombus formation was observed and images recorded over time. (A) Representative composite images of fluorescence and brightfield data depicting thrombus formation show fura-2-loaded platelet accumulation (green, yellow) and calcium mobilization (yellow). Pre, before injury. (B) Platelet accumulation during thrombus formation represented by the median integrated fluorescence intensity ( $y$ axis) of fura-2-labeled platelets excited at $380 \mathrm{~nm}$. Platelets were studied in the absence (upper curve) or presence (lower curve) of BAPTA-AM. F platelets, median integrated fluorescence of platelets, with excitation at $380 \mathrm{~nm}$ and emission at $510 \mathrm{~nm}$. (C) Representative composite images of fluorescence and bright-field data depicting thrombus formation show fura-2-loaded platelet accumulation (green, yellow) and calcium mobilization (yellow) in the presence of BAPTA-AM. (D) Calcium mobilization during thrombus formation depicted by the median integrated fluorescence intensity ( $y$ axis) of fura-2-labeled platelets excited at $340 \mathrm{~nm}$. Platelets were studied in the absence (upper curve) or presence (lower curve) of BAPTA$\mathrm{AM}$. These data represent the median of experiments performed in 18 thrombi from 3 mice. $\mathrm{F}$ calcein, median integrated fluorescence of the signal corresponding to calcein-labeled platelets.

induced in 3 mice, the median fura-2-loaded platelet accumulation (Figure 2B) and median calcium mobilization (Figure 2D) over time was calculated. Fura-2-loaded platelets rapidly accumulated at the site of vessel wall injury, then the thrombus decreased in size and the number of fura-2-loaded platelets diminished (Figure 2B). When fura-2-loaded platelets were treated with the calcium chelator BAPTA-AM prior to infusion into a donor mouse, calcium mobilization was inhibited (Figure 2C). BAPTA-AM-treated, fura-2-loaded platelets transiently bound to the developing thrombus (Figure 2B) and did not mobilize calcium (Figure 2D). These results indicate that platelet activation as monitored by calcium mobilization occurs in the developing thrombus in vivo in our thrombosis model. However, platelets are able to reversibly bind to the developing thrombus independent of or prior to calcium mobilization.

$v W F$ is important for platelet accumulation during thrombus formation in vivo. Spatial and temporal resolution of 2 features of initial thrombus development were determined using $v \mathrm{WF}^{-/-}$and WT mice. Platelet activation was monitored by calcium mobilization, and platelet accumulation on the vessel wall was monitored using a fluorescently labeled antibody directed against CD41 (12). In WT mice, platelets adhered and accumulated rapidly at the site of injury, contributing to the early phase of platelet accumulation.
The thrombus grew rapidly to its maximum size, then decreased in size (Figure 3A; ref. 13). $v \mathrm{WF}^{-/-}$mice showed similar kinetics of platelet accumulation (Figure 3A). Despite the absence of vWF, these mice had significant platelet thrombus formation, although the maximal size of thrombi was reduced compared with that in WT mice (Figure 3A). When the time to maximum thrombus size was compared in 43 thrombi in 4 WT mice and 39 thrombi in 4 $v W^{-1-}$ mice, no significant difference was observed (Figure 3B). However, a significant reduction $(P<0.001)$ in the size of thrombi was observed in $v \mathrm{WF}^{-/-}$mice in comparison with WT mice (Figure 3C). The largest thrombi observed in $v \mathrm{WF}^{-/-}$mice were not restricted to a single $\nu \mathrm{WF}^{-/-}$animal but were distributed among all of the $v \mathrm{WF}^{-1-}$ mice. This difference in thrombus size between WT and $v \mathrm{WF}^{-/-}$mice was confirmed by plotting the quartile distribution of thrombus size (Figure 3D). Thirty percent of the thrombi formed in $v \mathrm{WF}^{-1-}$ mice were present in the first quartile, representing the smallest thrombi, versus $20 \%$ for the WT mice. Only $18 \%$ of the thrombi formed in $v \mathrm{WF}^{-/-}$mice were distributed in the largest quartile, versus $34 \%$ for the WT mice. These results indicate that the maximal size of the thrombus was significantly reduced in $v \mathrm{WF}^{-1-}$ mice in comparison with WT mice. However, the kinetics of thrombus formation were similar. 


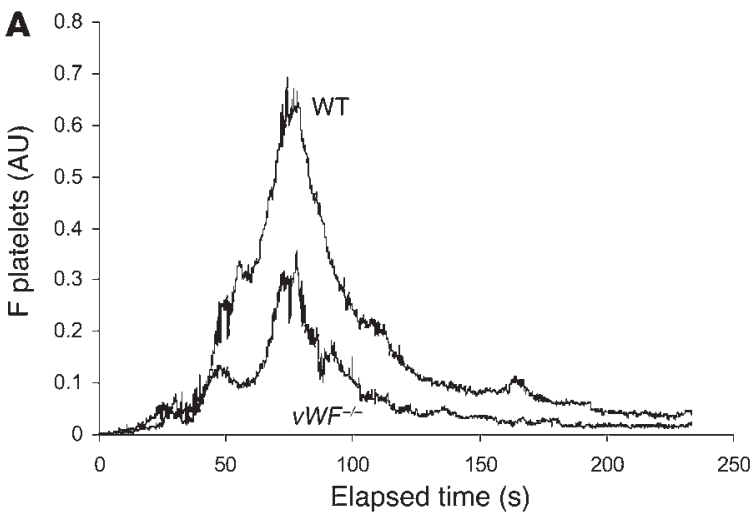

B
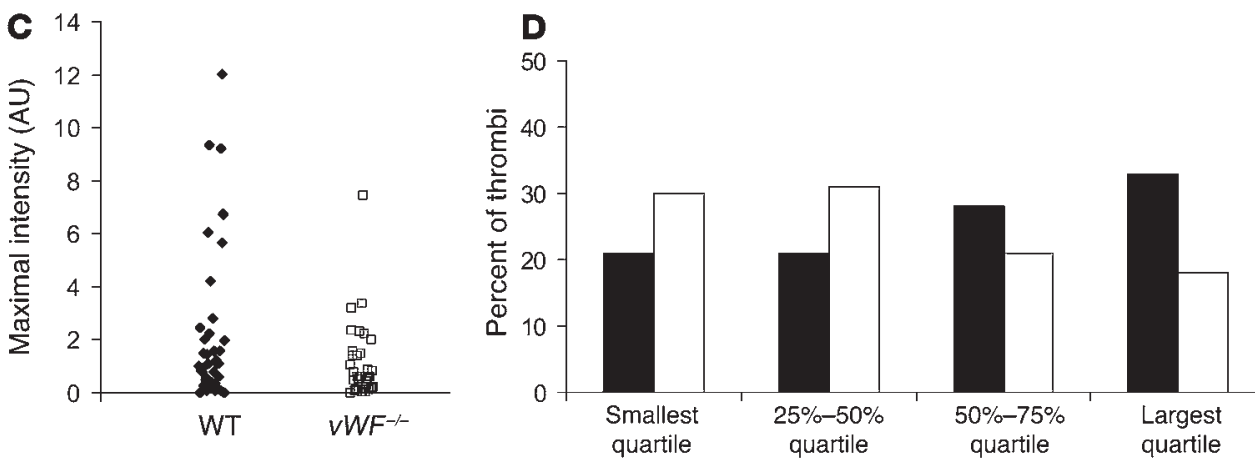

Figure 3

Platelet accumulation during thrombus formation after vessel wall injury in WT and $v W F^{-/-}$mice. Platelets were labeled with anti-mouse CD41 Fab fragments conjugated to Alexa Fluor 647. (A) The median integrated platelet fluorescence ( $y$ axis) for 43 thrombi in 4 WT mice and for 39 thrombi in $4 v W^{-1-}$ mice is presented versus time after vessel wall injury. (B) The distribution of the time to reach maximal size for each thrombus in WT mice and in $v W F^{-/-}$mice. No significant difference was observed by the Wilcoxon rank sum test. (C) The distribution of the integrated platelet fluorescence for each thrombus in WT and $v W F^{-1-}$ mice at maximal size. WT thrombi were significantly larger than $v W F^{-1-}$ thrombi by the Wilcoxon rank sum test $(P<0.001)$. (D) The quartile distribution of the maximal integrated platelet fluorescence for each thrombus in WT and $v W F^{-/-}$mice. Forty-three thrombi in WT mice and 39 thrombi in $v W F^{-/-}$mice were ranked, and the percentage of thrombi of each genotype was determined independently in each quartile of the rank order. Black bars, WT mice; white bars, vWF ${ }^{-1-}$ mice.

$\nu$ WF is not required for platelet activation during thrombus formation in vivo. Previous in vitro studies have described 2 calcium peaks per platelet when platelets in a flow chamber come in contact with immobilized vWF. The first peak is dependent on the interaction of platelets with $\mathrm{VWF}$ and has been interpreted as being an important signal for activation of $\alpha_{\mathrm{II}} \beta_{3}(11,14,15)$. To determine whether $\mathrm{vWF}$ is required in vivo for platelet activation - independent of its role in platelet adhesion and aggregation - platelet activation at the site of vascular injury was studied in $v \mathrm{WF}^{-1-}$ mice. Although platelet accumulation was attenuated in these mice (Figure 3A), platelet activation as monitored by calcium mobilization was observed in $v \mathrm{WF}^{-1-}$ mice (Figure 4A). Statistical analysis of the median fluorescence associated with calcium mobilization in multiple thrombi in $\mathrm{WT}$ and $v \mathrm{WF}^{-/-}$mice allowed quantitation of platelet activation in these mice (Figure 4B). To calculate calcium mobilization per platelet (Figure 5A), the median integrated fluorescence associated with activated platelets was compared with the median integrated fluorescence associated with the total platelets accumulated within the thrombus (Figure 5B). This approach was validated in in vitro experiments in which a calibration curve was established comparing the fluorescence associated with calcium mobilization divided by the total platelet fluorescence as a function of the percentage of platelets capable of undergoing calcium mobilization (Supplemental Figure 1; supplemental material available online with this article; doi:10.1172/JCI30537DS1). In separate in vivo experiments, a single fluorescent platelet was examined for calcium mobilization, thus allowing the direct calculation of calcium mobilization per platelet (data not shown). This measurement on individual platelets confirmed the results obtained by studying fluorescence of multiple platelets within the platelet thrombus. These experiments allowed us to draw the conclusion that platelet activation is equivalent in WT and $\nu \mathrm{WF}^{-/-}$mice in this thrombosis model.

GPVI is not required for calcium mobilization during thrombus formation following laser injury. We have previously demonstrated that platelet accumulation associated with laser-induced vessel wall injury under the conditions that we employ is not dependent upon either the presence of platelet GPVI or associated with the exposure of detectable collagen on the vessel wall (12). To determine whether GPVI is required for calcium mobilization, median platelet accumulation and calcium mobilization in WT and $F c R \gamma^{-/-}$mice deficient in GPVI were compared. Platelet activation as monitored by calcium mobilization was not dependent upon GPVI (Figures 5 and 6). Identical results were observed in WT mice depleted of GPVI using a monoclonal antibody directed against GPVI (data not shown).

Thrombin is required for calcium mobilization and platelet accumulation following laser injury. The requirement of thrombin for platelet activation leading to calcium mobilization was evaluated by treating WT mice with lepirudin, an inhibitor of thrombin. Fibrin generation and platelet accumulation were monitored within the developing thrombus using Alexa Fluor 647 conjugated to Fab fragments of antibodies against CD41 and Alexa Fluor 488 conjugated to fibrin-specific antibodies, respectively. Prior to infusion of lepirudin into a WT mouse, multiple thrombi were evaluated. After lepirudin infusion into the jugular vein of the mouse, multiple thrombi were again generated and fibrin and platelet accumulation quantitated. Under these conditions, fibrin generation was completely blocked and platelet accumulation was greatly attenuated in vivo by lepirudin (Figure 7). Calcium mobilization in fura-2-loaded WT platelets, infused into WT mice pretreated with lepirudin, was significantly reduced, although the initial phase of platelet accumulation was still present. Calcium mobilization 
A
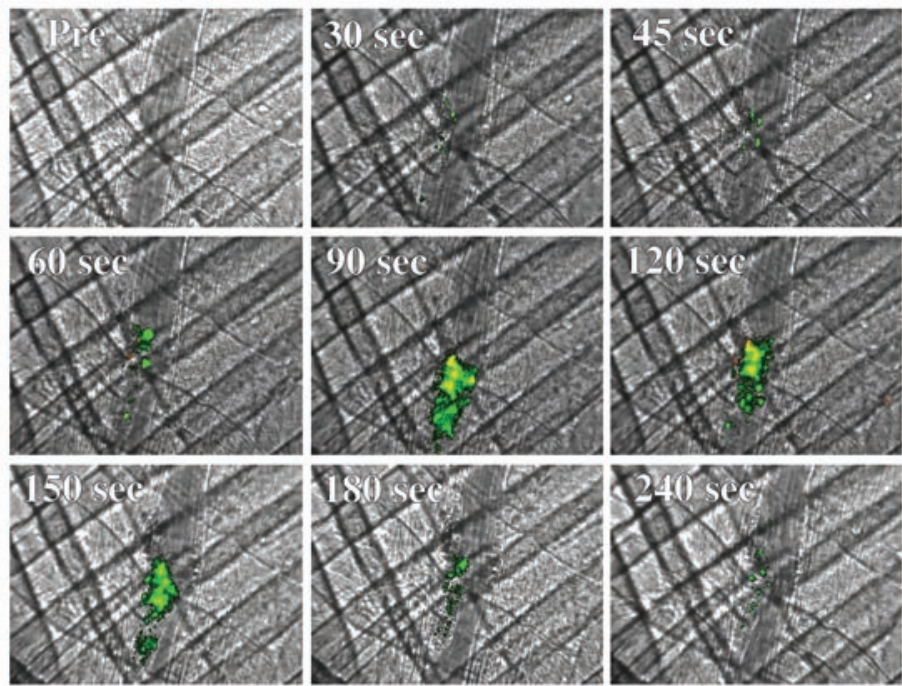

B

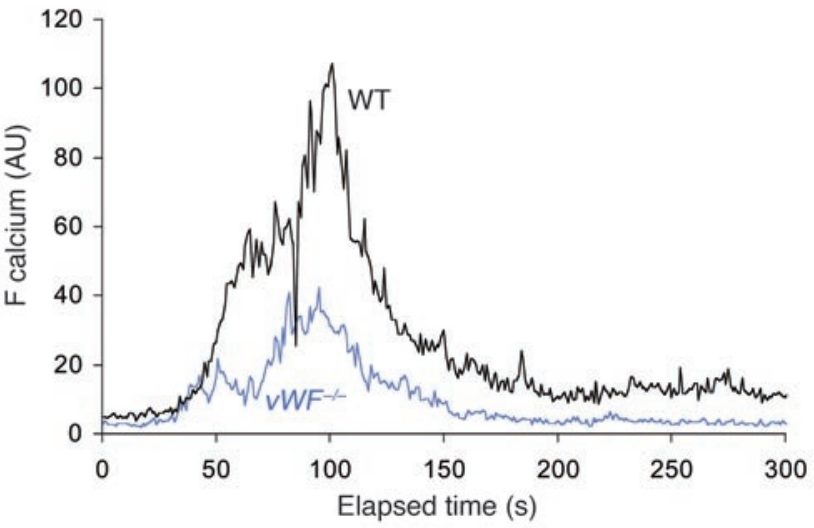

per platelet in WT mice treated with lepirudin was significantly reduced in comparison with that of untreated $\nu \mathrm{WF}^{-/-}, F c R \gamma^{-/-}$, and WT mice (Figure 5B). These results indicate that, in this in vivo thrombosis model under the conditions employed for laser injury, platelet activation is independent of vWF but dependent upon thrombin activity.

\section{Discussion}

In this study, we have separately monitored platelet activation and platelet accumulation in mice lacking vWF using laser-induced vessel wall injury, a thrombosis model that is dominated by tissue factor-mediated thrombin generation but in which platelet accumulation appears to be largely independent of GPVI and collagen under the conditions that we employ $(12,16)$. Our results indicate that platelet activation, as monitored directly by calcium mobilization, is normal in mice lacking vWF. We conclude that vWF

\section{Figure 5}

Calcium mobilization per platelet in WT mice, $v W F^{-/-}$mice, $F_{c} R \gamma^{-/-}$mice, and WT mice treated with lepirudin. In order to calculate calcium mobilization per platelet, the ratios of the signals corresponding to platelet accumulation and calcium mobilization (as assessed by the median integrated fluorescence intensity of each) were compared. (A) Platelet accumulation. (B) Calcium mobilization per platelet, presented as a ratio. $F_{C} R \gamma^{-1-}, F_{C} R \gamma^{-1-}$ mice lacking GPVI; WT + lepirudin, WT mice treated with lepirudin.

\section{Figure 4}

In vivo imaging of calcium mobilization during platelet activation during thrombus formation in $v W F^{-/-}$mice. (A) Platelets isolated from $v W F^{-/}$mice were loaded with fura-2/AM. Platelets $\left(250 \times 10^{6}\right.$ to $300 \times 10^{6}$ cells $)$ were infused into the circulation of a $v W F^{-/-}$mouse. Following laser-induced vessel wall injury, thrombus formation was observed and images recorded over time. (A) Representative composite images of fluorescence and bright-field data depicting thrombus formation show fura-2-loaded platelet accumulation (green, yellow) and calcium mobilization (yellow) in a $v W F^{-1-}$ mouse. (B) Calcium mobilization during thrombus formation represented by the median integrated fluorescence intensity ( $y$ axis) of fura-2-labeled platelets excited at $340 \mathrm{~nm}$. Black: WT mice; blue: $v W F^{-/-}$mice.

is not required for platelet activation during thrombus formation under circumstances in which the tissue factor-mediated pathway of thrombin generation is dominant. However, platelet accumulation is attenuated in the absence of vWF, as previously described (9). These results are unanticipated for 2 reasons. First, vWF has been shown to bind and activate platelets in in vitro flow studies, with the mechanical interaction of vWF and platelets leading to intracellular calcium mobilization (17). However, we find that in vivo, in the absence of significant collagen exposure and even at high shear, the docking function of vWF is not required for platelet activation. This is most likely due to intrinsic differences in the experimental design, since Mazzucato et al. (11) performed experiments in vitro with a high density of vWF adherent to the solid phase and in the presence of hirudin to block thrombin activity, whereas the current experiments were performed in a live mouse in the absence of anticoagulants, including thrombin inhibitors. Second, incorporation of a platelet into a thrombus in an injured vessel and platelet activation have been thought to occur simultaneously. However, we demonstrate that the platelet thrombus includes a large population of platelets that do not get

A

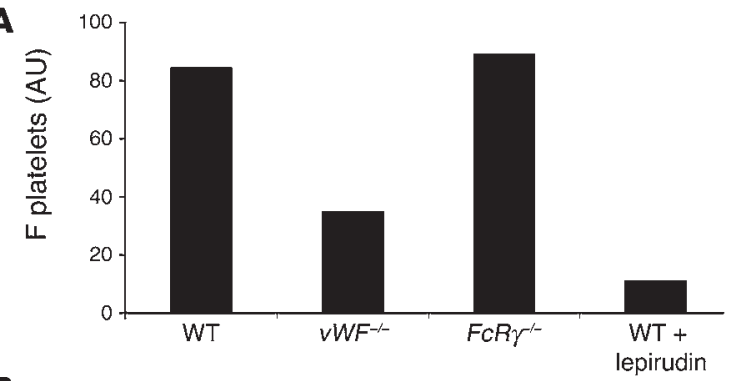

$\mathbf{B}$

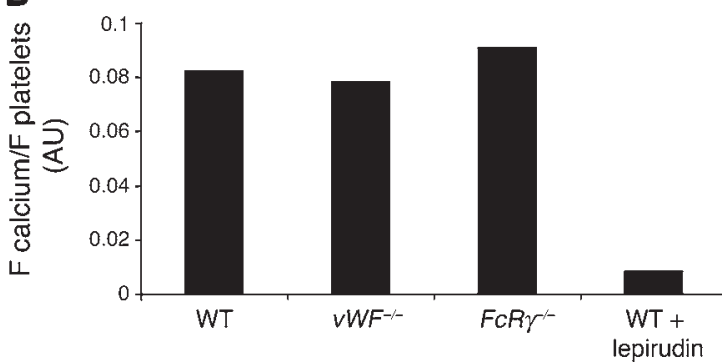


Pre
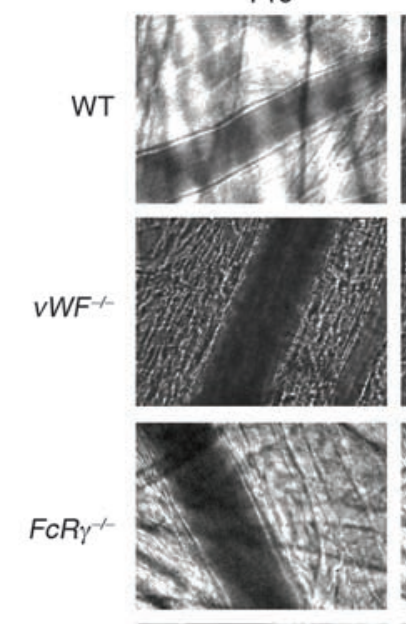

WT + lepirudin

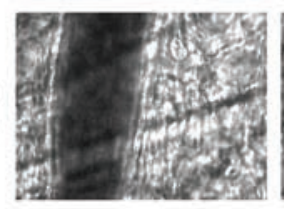

$30 \mathrm{~s}$
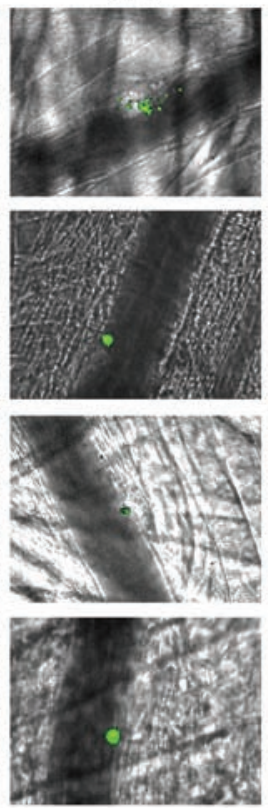

$60 \mathrm{~s}$
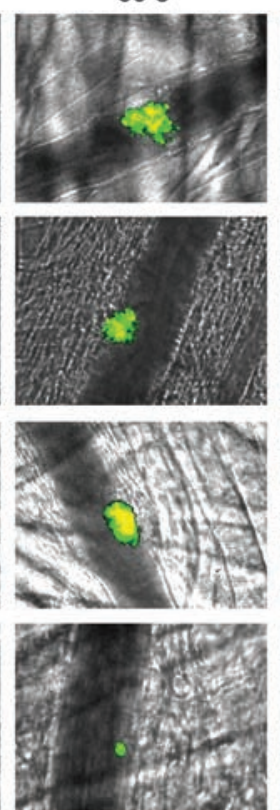

$90 \mathrm{~s}$
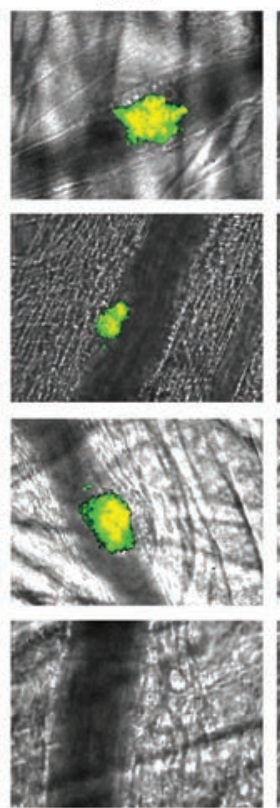

$120 \mathrm{~s}$
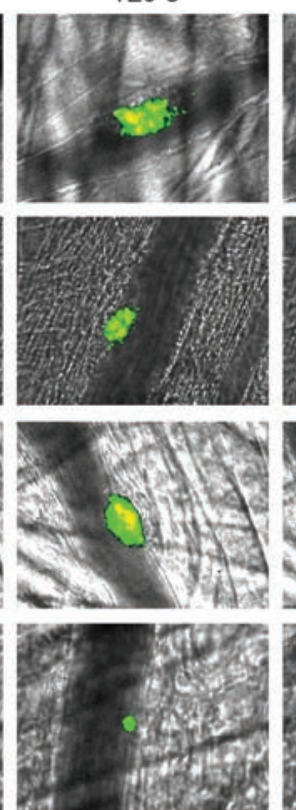

$150 \mathrm{~s}$
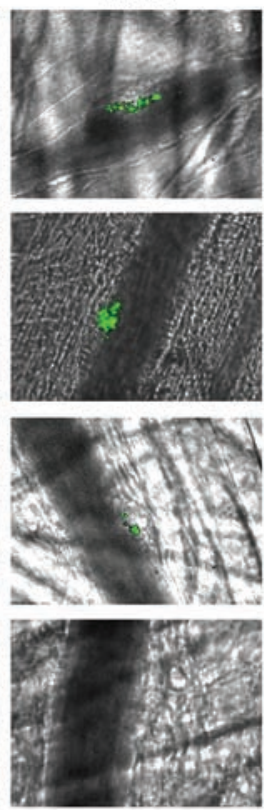

\section{Figure 6}

Role of thrombin, vWF, and GPVI in platelet accumulation and calcium mobilization. WT, vWF $F^{--}$, or FcR $\gamma^{-/}$platelets loaded with fura-2/AM were infused into a recipient mouse of the same genotype. In some experiments, the WT recipient mouse was treated with lepirudin. Thrombus formation was performed as described in Figure 2 and fura-2-loaded platelets imaged for platelet accumulation and calcium mobilization. Representative images were obtained before injury (pre) and 30,60, 90, 120, and 150 seconds after injury.

activated and that remain transiently bound to the developing thrombus. This point is clearly established by the large number of unactivated platelets (green) in the images of a developing thrombus in Figure 2A depicting platelet accumulation.

Central to platelet activation is the mobilization of intracellular calcium. Cytosolic $\mathrm{Ca}^{2+}$ levels rise from basal levels of approximately $50-120 \mathrm{nM}$ in unstimulated platelets to micromolar levels in activated platelets, depending upon the potency of the agonist. As a second messenger, calcium mobilization mediates many platelet functions, such as shape change, platelet secretion, and activation of various integrins residing on the platelet membrane $(18,19)$. In vitro studies under static or flow conditions have identified the intracellular pathways leading to the mobilization of intracellular calcium after platelet adhesion. In a static model, thrombin induces calcium mobilization within platelets, leading to the activation of specific integrins and secretion of platelet granules. In perfusion experiments where flow is adjusted to arterial shear rates, adhesion of platelets first requires the binding of the GPIb-IX-V complex on the surface of platelets to $\mathrm{vWF}(1,5,20,21)$. This interaction leads to sequential elevations in $\left[\mathrm{Ca}^{2+}\right]_{i}$. The first $(\alpha / \beta)$ peak, associated with mechanical stimulation and rapid $\mathrm{Ca}^{2+}$ release from intracellular stores, is coupled to a first stage of $\alpha_{\text {II }} \beta_{3}$ activation $(11,15,22)$. Subsequent binding of activated $\alpha_{\mathrm{II}} \beta_{3}$ to $\mathrm{VWF}$ and fibrinogen leads to a second $\mathrm{Ca}^{2+}$ elevation ( $\gamma$ peak) of greater amplitude and duration that is necessary for the stable activation of integrins and irreversible adhesion and aggregation of platelets (23). Last, continuous P2Y1 and P2Y12 stimulation resulting in cyclic $\left[\mathrm{Ca}^{2+}\right]_{i}$ oscillations appears to be necessary for maintaining the state of activation of $\alpha_{\text {III }} \beta_{3}$ required for thrombus stability in flowing blood (24).

We adapted a well-developed technique for monitoring calcium transients in live cells to follow calcium mobilization in platelets during thrombus formation in a living mouse. Although we are not able to quantitate intracellular calcium concentrations in cells in the circulation by ratio imaging (25), we established what we believe to be a novel method for monitoring platelet activation in vivo. We can measure platelet activation in vivo with high spatial and temporal resolution, providing new insights into this process during thrombus formation in a living mouse. For example, using calcium mobilization as a reporter of platelet activation, we demonstrate that $\mathrm{vWF}$ is not required for calcium mobilization. Furthermore, we observed transient adhesion of BAPTA-AM-treated platelets to a developing thrombus, indicating that the tethering of platelets to a developing thrombus is independent of calcium mobilization in a living mouse.

Our in vivo experiments on arterioles within the cremaster muscle microcirculation were performed at locations where shear rates were approximately $1,500 \mathrm{~s}^{-1}$. Under these conditions, human platelet thrombus formation is vWF dependent. Although mouse platelets are smaller, albeit more numerous, our in vivo experiments (Figure 3A) as well as those of others $(6,9,26)$ indicate that, under these conditions, platelet thrombus formation is also $\mathrm{vWF}$ dependent in mouse cremaster and mesentery arterioles.

Previous studies of thrombus formation in arterioles of $v \mathrm{WF}^{-1-}$ mice employed the ferric chloride model of vascular injury (9), another thrombosis model in which thrombus formation requires GPVI expression and exposure of collagen in the subendothelial matrix. Our current results suggest that the role of vWF in platelet activation may be limited to the collagen-platelet GPVI pathway, where vWF interaction with platelets is critical. However, as shown in the current study, platelet activation, when initiated by thrombin, the end product of the tissue factor-mediated pathway, is independent of vWF. This concept would be completely 

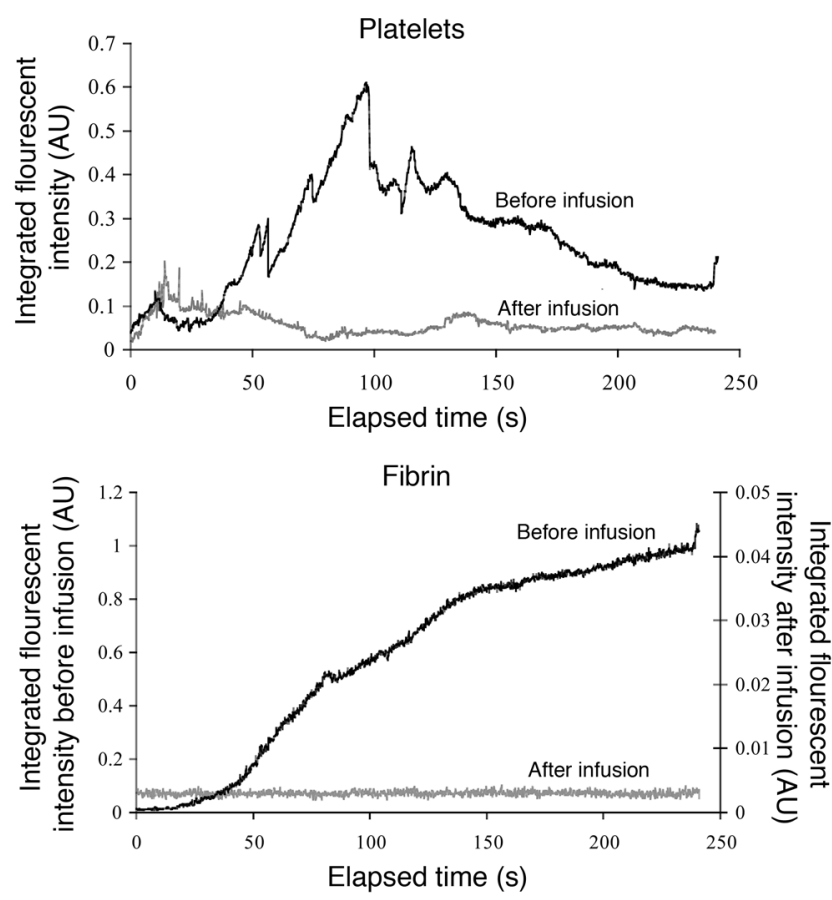

consistent with the observations that von Willebrand disease is not fully protective from thromboembolic disease, particularly atherothrombosis, since these diseases are characterized by an important role for thrombin.

\section{Methods}

Mice. WT C57BL/6J, $v \mathrm{WF}^{-1-}$, and $F c R \gamma^{-/-}$mice were obtained from The Jackson Laboratory. The characteristics of the $v \mathrm{WF}^{-/-}$and $F c R \gamma^{-/-}$mice have been previously described (9). In all of the mouse experiments in the studies reported here, donor platelets were obtained from mice of the same genotype as the recipient mouse. The Beth Israel Deaconess Medical Center Institutional Animal Care and Use Committee approved all animal care and experimental procedures.

Antibodies and reagents. Rat anti-mouse CD41 antibody (clone MWReg30) and rat anti-mouse P-selectin antibody (clone RB 40.34) were from BD Biosciences - Pharmingen. Mouse anti-human fibrin II $\beta$-chain antibody (clone NYBT2G1) was from Accurate. Fab fragments from the anti-CD41 antibody were generated using the ImmunoPure Fab Preparation Kit from Pierce and then conjugated to Alexa Fluor 647 according to the manufacturer's instructions (Invitrogen). Anti-fibrin antibody was conjugated to Alexa Fluor 488. Lepirudin (Berlex) was infused in vivo every 30 minutes via a jugular catheter at a dose of $8 \mathrm{U} / \mathrm{g}$ mouse.

Preparation and pretreatment of washed platelets. Blood was collected from mice in a citrate solution (ACD: $85 \mathrm{mM}$ trisodium citrate, $67 \mathrm{mM}$ citric acid, $111.5 \mathrm{mM}$ glucose, $\mathrm{pH} 4.5$ ) in the presence of $0.5 \mathrm{mM}$ prostacyclin ( $\mathrm{PGI}_{2}$; Calbiochem-Novabiochem) and $0.02 \mathrm{U} / \mathrm{ml}$ apyrase (Sigma-Aldrich). Platelets were separated from freshly drawn blood by centrifugation and washed twice in CGS buffer (13 mM trisodium citrate, $30 \mathrm{mM}$ dextrose, and $120 \mathrm{mM} \mathrm{NaCl}, \mathrm{pH} 7.0$ ) in the presence of $0.04 \mathrm{U} / \mathrm{ml}$ apyrase and 500 $\mathrm{nM} \mathrm{PGI} 2$, as previously described (9). Washed platelets were resuspended at a concentration of $1 \times 10^{8}$ platelets $/ \mathrm{ml}$ in Tyrode buffer $(138 \mathrm{mM} \mathrm{NaCl}, 2.9$ $\mathrm{mM} \mathrm{KCl}, 12 \mathrm{mM} \mathrm{NaHCO} 3,0.36 \mathrm{mM} \mathrm{NaH}_{2} \mathrm{PO}_{4}, 5.5 \mathrm{mM}$ glucose, $1.8 \mathrm{mM}$ $\mathrm{CaCl}_{2}$, and $0.4 \mathrm{mM} \mathrm{MgCl}_{2}, \mathrm{pH} 7.4$ ) containing $0.2 \% \mathrm{BSA}$ (Sigma-Aldrich). Platelets were incubated with $1 \mu \mathrm{g} / \mathrm{ml}$ of red-orange calcein AM (Molecular Probes; Invitrogen) or $3 \mu \mathrm{M}$ fura-2/AM (Molecular Probes; Invitrogen) in

\section{Figure 7}

Effect of lepirudin on platelet accumulation and fibrin deposition in WT mice. Thrombus formation was induced as described in Figure 1, and platelets and fibrin detected using Fab fragments of antibodies against CD41 and fibrin-specific antibodies, respectively. The median integrated fluorescence over time is presented for 30 thrombi generated in 3 WT mice. After generating a series of 10 thrombi per mouse, lepirudin ( $8 \mathrm{U} / \mathrm{g}$ mouse) was infused and 9-10 additional thrombi generated per mouse. A total of 28 thrombi before infusion and 29 thrombi after infusion were studied in 3 mice. Black, before infusion; gray, after infusion.

the absence or presence of $50 \mu \mathrm{M}$ BAPTA-AM (Calbiochem-Novabiochem) for 40 minutes in the dark at $37^{\circ} \mathrm{C}$, centrifuged, and resuspended in Tyrode buffer at $100 \times 10^{7}$ platelets $/ \mathrm{ml}$ before injection into a recipient mouse.

Flow cytometry. Resting or thrombin-activated $(0.1 \mathrm{U} / \mathrm{ml})$ platelets $\left(1 \times 10^{6}\right.$ cells) were incubated with $2.5 \mu \mathrm{g}$ of FITC-labeled antibody directed against mouse P-selectin (clone RB40.34) for 5 minutes. Expression of P-selectin on the surface of resting and activated platelets was analyzed using a BD FACSCalibur flow cytometer. Flow cytometry experiments were performed on $1.5 \times 10^{7}$ washed platelets.

Intravital microscopy. Intravital videomicroscopy of the cremaster muscle microcirculation was done as previously described (27). Mice were preanesthetized with intraperitoneal ketamine (125 mg/kg; Abbott), xylazine $(12.5 \mathrm{mg} / \mathrm{kg}$; Phoenix Pharmaceuticals Inc.), and atropine $(0.25 \mathrm{mg} / \mathrm{kg}$; American Pharmaceutical Partners). A tracheal tube was inserted and the mouse maintained at $37^{\circ} \mathrm{C}$ on a thermo-controlled rodent blanket. To maintain anesthesia, Nembutal (Abbott) was administered through a cannulus placed in the jugular vein. After the scrotum was incised, the testicle and surrounding cremaster muscle were exteriorized onto an intravital microscopy tray. The cremaster preparation was superfused with thermocontrolled $\left(36^{\circ} \mathrm{C}\right)$ and aerated $\left(95 \% \mathrm{~N}_{2}, 5 \% \mathrm{CO}_{2}\right)$ bicarbonate-buffered saline throughout the experiment. Microvessel data were obtained using an Olympus AX microscope with a $60 \times 0.9$-numerical aperture water immersion objective. The digital wide-field fluorescence microscopy system has previously been described (27). Digital images were captured with a Cooke Sensicam CCD camera in $640 \times 480$ format.

Laser-induced injury. Exogenous labeled platelets $\left(250 \times 10^{6}\right.$ to $\left.300 \times 10^{6}\right)$ were infused into the circulation of an anesthetized mouse. When employed, lepirudin (Berlex) was injected via a jugular catheter at a dose of $8 \mathrm{U} / \mathrm{g}$ mouse 5-15 minutes prior to laser-induced injury. Vessel wall injury was induced with a Micropoint Laser System (Photonics Instruments) focused through the microscope objective, parfocal with the focal plane and aimed at the vessel wall, as previously described (12). Typically 1 or 2 pulses were required to induce vessel wall injury. The severity of injury was comparable to that in the laser injury models employed by others but significantly distinct from the superficial or moderate injuries used by the 
same research groups $(28,29)$. For in vivo calcium mobilization experiments, the number of thrombi formed was limited to 6 over a 45 -minute period. Multiple thrombi were studied in a single mouse, with new thrombi formed upstream of earlier thrombi to avoid any contribution from thrombi generated earlier in the animal under study. There were no characteristic trends in thrombus size or thrombus composition in sequential thrombi generated in a single mouse during an experiment. Image analysis was performed using Slidebook 4.1 (Intelligent Imaging Innovations). Fluorescence data were captured digitally at up to 50 frames per second and analyzed as previously described (27). The kinetics of thrombus formation were analyzed by determining median fluorescence values over time in approximately 30 thrombi (30).

Statistics. Statistical analyses were performed using the Wilcoxon rank test.

1. Ruggeri, Z.M. 2002. Platelets in atherothrombosis. Nat. Med. 8:1227-1234.

2. Gibbins, J.M. 2004. Platelet adhesion signalling and the regulation of thrombus formation. J. Cell Sci. 117:3415-3425.

3. Furie, B., and Furie, B.C. 2005. Thrombus formation in vivo. J. Clin. Invest. 115:3355-3362. doi:10.1172/ JCI26987.

4. Jackson, S.P., Nesbitt, W.S., and Kulkarni, S. 2003. Signaling events underlying thrombus formation. J. Thromb. Haemost. 1:1602-1612.

5. Savage, B., Saldivar, E., and Ruggeri, Z.M. 1996. Initiation of platelet adhesion by arrest onto fibrinogen or translocation on von Willebrand factor. Cell. 84:289-297.

6. Maxwell, M.J., et al. 2006. Identification of a two-stage platelet aggregation process mediating shear-dependent thrombus formation. Blood. 109:566-575.

7. Ikeda, Y., et al. 1993. Transmembrane calcium influx associated with von Willebrand factor binding to GP Ib in the initiation of shear-induced platelet aggregation. Thromb. Haemost. 69:496-502.

8. Kasirer-Friede, A., et al. 2004. Signaling through GP Ib-IX-V activates $\alpha$ IIb $\beta 3$ independently of other receptors. Blood. 103:3403-3411.

9. Denis, C., et al. 1998. A mouse model of severe von Willebrand disease: defects in hemostasis and thrombosis. Proc. Natl. Acad. Sci. U. S. A. 95:9524-9529.

10. Ni, H., et al. 2000. Persistence of platelet thrombus formation in arterioles of mice lacking both von Willebrand factor and fibrinogen. J. Clin. Invest. 106:385-392

11. Mazzucato, M., Pradella, P., Cozzi, M.R., De Marco, L., and Ruggeri, Z.M. 2002. Sequential cytoplasmic calcium signals in a 2 -stage platelet activation process induced by the glycoprotein Ibalpha mechanoreceptor. Blood. 100:2793-2800.

12. Dubois, C., Panicot-Dubois, L., Merrill-Skoloff, G.,
Furie, B., and Furie, B.C. 2006. Glycoprotein VIdependent and -independent pathways of thrombus formation in vivo. Blood. 107:3902-3906.

13. Chou, J., et al. 2004. Hematopoietic cell-derived microparticle tissue factor contributes to fibrin formation during thrombus propagation. Blood. 104:3190-3197.

14. Nesbitt, W.S., et al. 2002. Distinct glycoprotein $\mathrm{Ib} / \mathrm{V} / \mathrm{IX}$ and integrin alpha IIbbeta 3-dependent calcium signals cooperatively regulate platelet adhesion under flow. J. Biol. Chem. 277:2965-2972.

15. Mazzucato, M., Cozzi, M.R., Pradella, P., Ruggeri, Z.M., and De Marco, L. 2004. Distinct roles of ADP receptors in von Willebrand factor-mediated platelet signaling and activation under high flow. Blood. 104:3221-3227.

16. Vandendries, E., Hamilton, J.R., Coughlin, S.R., Furie, B., and Furie, B.C. 2007. Par4 is required for platelet thrombus propagation but not fibrin generation in a mouse model of thrombosis. Proc. Natl. Acad. Sci. U. S. A. 104:288-292.

17. Mazzucato, M., et al. 1998. Characterization of the initial alpha-thrombin interaction with glycoprotein Ib alpha in relation to platelet activation. J. Biol. Chem. 273:1880-1887.

18. Sargeant, P., and Sage, S.O. 1994. Calcium signalling in platelets and other nonexcitable cells. Pharmacol. Ther. 64:395-443.

19. Rink, T.J., and Sage, S.O. 1990. Calcium signaling in human platelets. Annu. Rev. Physiol. 52:431-449.

20. Alevriadou, B.R., et al. 1993. Real-time analysis of shear-dependent thrombus formation and its blockade by inhibitors of von Willebrand factor binding to platelets. Blood. 81:1263-1276.

21. Savage, B., Almus-Jacobs, F., and Ruggeri, Z.M. 1998. Specific synergy of multiple substrate-receptor interactions in platelet thrombus formation under flow. Cell. 94:657-666.
22. Nesbitt, W.S., et al. 2003. Intercellular calcium communication regulates platelet aggregation and thrombus growth. J. Cell Biol. 160:1151-1161.

23. Kuwahara, M., Sugimoto, M., Tsuji, S., Miyata, S., and Yoshioka, A. 1999. Cytosolic calcium changes in a process of platelet adhesion and cohesion on a von Willebrand factor-coated surface under flow conditions. Blood. 94:1149-1155.

24. Goto, S., Tamura, N., Ishida, H., and Ruggeri, Z.M. 2006. Dependence of platelet thrombus stability on sustained glycoprotein IIb/IIIa activation through adenosine $5^{\prime}$-diphosphate receptor stimulation and cyclic calcium signaling. J. Am. Coll. Cardiol. 47:155-162.

25. Pozzan, T., Lew, D.P., Wollheim, C.B., and Tsien, R.Y. 1983. Is cytosolic ionized calcium regulating neutrophil activation? Science. 221:1413-1415.

26. Bergmeier, W., et al. 2006. The role of platelet adhesion receptor GPIb $\alpha$ far exceeds that of its main ligand, von Willebrand factor, in arterial thrombosis. Proc. Natl. Acad. Sci. U. S. A. 103:16900-16905.

27. Falati, S., Gross, P., Merrill-Skoloff, G., Furie, B.C., and Furie, B. 2002. Real-time in vivo imaging of platelets, tissue factor and fibrin during arterial thrombus formation in the mouse. Nat. Med. 8:1175-1181.

28. Nonne, C., et al. 2005. Importance of platelet phospholipase Cgamma2 signaling in arterial thrombosis as a function of lesion severity. Arterioscler. Thromb. Vasc. Biol. 25:1293-1298.

29. Mangin, P., et al. 2006. Thrombin overcomes the thrombosis defect associated with platelet GPVI/ FcRgamma deficiency. Blood. 107:4346-4353.

30. Dubois, C., Atkinson, B., Furie, B.C., and Furie, B. 2007. Real-time in vivo imaging of platelets during thrombus formation. In Platelets. A.D. Michelson, editor. Academic Press. Amsterdam, The Netherlands. 611-626. 